PROCEEDINGS OF THE

AMERICAN MATHEMATICAL SOCIETY

Volume 131, Number 7 , Pages 2171-2179

S 0002-9939(02)06777-1

Article electronically published on December 30, 2002

\title{
EXTENDED CESÀRO OPERATORS ON MIXED NORM SPACES
}

\author{
ZHANGJIAN HU
}

(Communicated by Joseph A. Ball)

\begin{abstract}
We define an extended Cesàro operator $T_{g}$ with holomorphic symbol $g$ in the unit ball $B$ of $C^{n}$ as$$
T_{g}(f)(z)=\int_{0}^{1} f(t z) \Re g(t z) \frac{d t}{t}, \quad f \in H(B), z \in B,
$$

where $\Re g(z)=\sum_{j=1}^{n} z_{j} \frac{\partial f}{\partial z_{j}}$ is the radial derivative of $g$. In this paper we characterize those $g$ for which $T_{g}$ is bounded (or compact) on the mixed norm space $H_{p, q}(w)$.
\end{abstract}

\section{INTRODUCTION}

We denote by $D$ the unit disk in the complex plane $C$. For a holomorphic function $f(z)$ on $D$ with Taylor expansion $f(z)=\sum_{j=0}^{\infty} a_{j} z^{j}$, the Cesàro operator acting on $f$ is

$$
C[f](z)=\sum_{j=0}^{\infty}\left(\frac{1}{j+1} \sum_{k=0}^{j} a_{k}\right) z^{j} .
$$

It is well known that $C[\cdot]$ acts as a bounded linear operator on various spaces of holomorphic functions (see 7], 8], 13], 15]) including the Hardy and Bergman spaces. In particular $C\left[H^{p}\right] \subset H^{p}$ for each $p \in(0, \infty)$. In [11 Shi and Ren proved $C[\cdot]$ is bounded on the mixed norm space.

A little calculation shows $C[f](z)=\frac{1}{z} \int_{0}^{z} f(t)\left(\log \frac{1}{1-t}\right)^{\prime} d t$. Hence, on most holomorphic function spaces, $C[f]$ is bounded if and only if the integral operator $f \mapsto \int_{0}^{z} f(t)\left(\log \frac{1}{1-t}\right)^{\prime} d t$ is bounded. From this point of view it is natural to consider the extended Cesàro operator $T_{g}$ with holomorphic symbol $g$

$$
T_{g} f(z)=\int_{0}^{z} f(t) g^{\prime}(t) d t .
$$

The boundedness of $T_{g}$ on Hardy space and Bergman space are studied in [1] and [2].

Received by the editors May 8, 2001 and, in revised form, February 26, 2002. 2000 Mathematics Subject Classification. Primary 47B38, 32A36.

Key words and phrases. Cesàro operator, mixed norm space, normal weight.

This research was partially supported by the 151 Projection and the Natural Science Foundation of Zhejiang Province. 
The purpose of this paper is to define the extended Cesàro operator on the unit ball $B$ of $C^{n}$ and to characterize those holomorphic symbols on $B$ for which the induced operator is bounded (or compact) on mixed norm space. Our work will extend [11, 15].

The class of all holomorphic functions on the unit ball $B$ of $C^{n}$ will be denoted by $H(B)$. For $g \in H(B)$ having the homogeneous expansion $g=\sum_{j=0}^{\infty} G_{j}$, as in [9] we let $\Re g(z)=\sum_{j=0}^{\infty} j G_{j}(z)$ be the radial derivative of $g$. With a little calculation one can show that $\Re g(z)=\sum_{j=1}^{n} z_{j} \frac{\partial g}{\partial z_{j}}$. Given $g \in H(B)$, the extended Cesàro operator $T_{g}$ with symbol $g$ is defined on $H(B)$ as

$$
T_{g}(f)(z)=\int_{0}^{1} f(t z) \Re g(t z) \frac{d t}{t}, \quad f \in H(B), z \in B .
$$

It is trivial that when $n=1,(2)$ is just (1).

A positive continuous function $\varphi$ on $[0,1)$ is called normal if there are two constants $b>a>0$ such that

$$
\frac{\varphi(r)}{(1-r)^{a}} \downarrow 0, \quad \frac{\varphi(r)}{(1-r)^{b}} \uparrow \infty
$$

as $r \rightarrow 1^{-}$. Given $\varphi$ normal, for $f \in H(B)$ we set

$$
\|f\|_{p, q, \varphi}=\left\{\int_{0}^{1} M_{q}^{p}(f, r) \frac{\varphi^{p}(r)}{1-r} d r\right\}^{\frac{1}{p}}, \quad 0<p<\infty
$$

and

$$
\|f\|_{\infty, q, \varphi}=\sup _{0<r<1} M_{q}(f, r) \varphi(r) .
$$

Here

$$
\begin{gathered}
M_{q}(f, r)=\left\{\int_{\partial B}|f(r \zeta)|^{q} d \sigma(\zeta)\right\}^{\frac{1}{q}}, \quad 0<q<\infty, \\
M_{\infty}(f, r)=\sup _{\zeta \in \partial B}|f(r \zeta)| .
\end{gathered}
$$

The mixed norm space $H_{p, q}(\varphi), 0<p, q \leq \infty$, consists of all $f \in H(B)$ such that $\|f\|_{p, q, w}<\infty$. When $0<p=q<\infty, H_{p, q}(\varphi)$ is just the weighted Bergman space

$$
A_{a}^{p}(\varphi)=\left\{f \in H(B):\|f\|_{A_{a}^{p}}=\left\{\int_{B}|f(z)|^{p^{p}} \frac{\varphi^{p}(|z|)}{1-|z|} d m(|z|)\right\}^{\frac{1}{p}}<\infty\right\} .
$$

As in [14] a function $f \in H(B)$ is called a Bloch function if $\sup \left\{Q_{f}: z \in B\right\}<\infty$, and $f$ is called a little Bloch function if $\lim _{|z| \rightarrow 1} Q_{f}(z)=0$. By Theorem 1.8 and Corollary 3.8 in [14] we know that a holomorphic function $f$ is a Bloch function if and only if

$$
\|f\|_{\mathcal{B}}=\sup \left\{|\Re f(z)|\left(1-|z|^{2}\right): z \in B\right\}<\infty,
$$

and $f$ is a little Bloch function if and only if

$$
\lim _{|z| \rightarrow 1}|\Re f(z)|\left(1-|z|^{2}\right)=0 .
$$

The sets of all Bloch and little Bloch functions will be denoted by $\mathcal{B}$ and $\mathcal{B}_{0}$, respectively. We also know that $\|f\|_{\mathcal{B}}$ is an equivalent norm on $\mathcal{B} / C$. 
In what follows $C, C_{1}, C_{2}$ will stand for positive constants whose value may change from line to line but not depend on the functions in $H(B)$. The expression $A \simeq B$ means $C_{1} A \leq B \leq C_{2} A$.

Our main result is the following.

Theorem 1. Let $0<p, q \leq \infty$, and let $\varphi$ be normal. Then for $g \in H(B)$

(I) $T_{g}$ is bounded on $H_{p, q}(\varphi)$ if and only if $g \in \mathcal{B}$. Moreover, when $T_{g}$ is bounded,

$$
\left\|T_{g}\right\| \simeq\|g\|_{\mathcal{B}}
$$

(II) $T_{g}$ is compact on $H_{p, q}(\varphi)$ if and only if $g \in \mathcal{B}_{0}$.

\section{Equivalent NORMS ON $H_{p, q}$}

Recall that, for $f \in H(B)$, the radial derivative of $f$ can be expressed as

$$
\Re f(z)=\sum_{j=1}^{n} z_{j} \frac{\partial f(z)}{\partial z_{j}} .
$$

For $m=1,2, \cdots$, write $\Re^{m} f(z)=\Re\left(\Re^{m-1} f(z)\right), \operatorname{grad}_{m} f(z)\left|=\sum_{|\alpha|=m}\right| \frac{\partial^{\alpha} f}{\partial z^{\alpha}} \mid$.

Lemma 1. Suppose $f \in H(B), f(0)=0$. Then

$$
\begin{gathered}
M_{q}(f, r) \leq \frac{C}{r} \int_{0}^{r} M_{q}(\Re f, t) d t, \quad \text { if } 1 \leq q \leq \infty, \\
M_{q}(f, r) \leq \frac{C}{r}\left\{\int_{0}^{r}(r-t)^{q-1} M_{q}^{q}(\Re f, t) d t\right\}^{\frac{1}{q}}, \quad \text { if } 0<q<1 .
\end{gathered}
$$

Proof. For $f \in H(B), f(0)=0$, and $z \in B$ we know

$$
f(z)=\int_{0}^{1} \frac{\Re f(t z)}{t} d t
$$

By Proposition 1.4.7 of [10],

$$
\begin{aligned}
\int_{\partial B}\left|\frac{\Re f(\operatorname{tr} \zeta)}{t}\right|^{q} d \sigma(\zeta) & =\int_{\partial B} d \sigma(\zeta) \int_{0}^{2 \pi}\left|\frac{\Re f\left(\left(t e^{i \theta}\right) r \zeta\right)}{t}\right|^{q} \frac{d \theta}{2 \pi} \\
& =\int_{\partial B} d \sigma(\zeta) \int_{0}^{2 \pi}\left|\frac{\Re f\left(\left(t e^{i \theta}\right) r \zeta\right)}{t e^{i \theta}}\right|^{q} \frac{d \theta}{2 \pi} .
\end{aligned}
$$

Then we know $\int_{\partial B}\left|\frac{\Re f(\operatorname{tr\zeta })}{t}\right|^{q} d \sigma(\zeta)$ is increasing with $t \in[0,1)$, because $\Re f(\operatorname{wr} \zeta) / w$ is a holomorphic function of $w$ in the unit disc $D$. Now for $1 \leq q \leq \infty$, by (5) and Minkowski's inequality,

$$
\begin{aligned}
M_{q}(f, r) & \leq \int_{0}^{1} d t\left\{\int_{\partial B}\left|\frac{\Re f(\operatorname{tr} \zeta)}{t}\right|^{q} d \sigma(\zeta)\right\}^{\frac{1}{q}} \\
& \leq C \int_{\frac{1}{2}}^{1} d t\left\{\int_{\partial B}\left|\frac{\Re f(\operatorname{tr} \zeta)}{t}\right|^{q} d \sigma(\zeta)\right\}^{\frac{1}{q}} \\
& \leq C \int_{0}^{1} d t\left\{\int_{\partial B}|\Re f(\operatorname{tr} \zeta)|^{q} d \sigma(\zeta)\right\}^{\frac{1}{q}} \\
& =C \frac{1}{r} \int_{0}^{r} M_{q}(\Re f, t) d t .
\end{aligned}
$$


To deal with the case $0<q<1$ we denote $t_{j}=1-2^{-j}, j=0,1,2, \cdots$. From [3] (or Lemma 2 in [10]) we obtain

$$
\int_{\partial B} \sup _{t_{j-1} \leq t \leq t_{j}}\left|\frac{\Re f(t r \zeta)}{t}\right|^{q} d \sigma(\zeta) \leq C \int_{\partial B}\left|\frac{\Re f\left(t_{j} r \zeta\right)}{t_{j}}\right|^{q} d \sigma(\zeta) .
$$

Then by (5) and [4, p. 57],

$$
\begin{aligned}
M_{q}^{q}(f, r) & \leq C \sum_{j=1}^{\infty} 2^{-j q} \int_{\partial B} \sup _{t_{j-1} \leq t \leq t_{j}}\left|\frac{\Re f(\operatorname{tr} \zeta)}{t}\right|^{q} d \sigma(\zeta) \\
& \leq C \sum_{j=1}^{\infty} 2^{-j q} \int_{\partial B}\left|\frac{\Re f\left(t_{j} r \zeta\right)}{t_{j}}\right|^{q} d \sigma(\zeta) \\
& \leq C \int_{0}^{1}(1-t)^{q-1} M_{q}^{q}(\Re f, r t) d t \\
& \leq C \frac{1}{r^{q}} \int_{0}^{r}(r-t)^{q-1} M_{q}^{q}(\Re f, t) d t .
\end{aligned}
$$

The lemma is proved.

The following theorem, which has its own interest, will be used in the proof of the main result.

Theorem 2. Let $0<p, q \leq \infty$ and let $m$ be a positive integer. Then for $f \in H(B)$,

$$
\|f\|_{p, q, \varphi} \simeq \sum_{j=0}^{m-1}\left|\operatorname{grad}_{j} f(0)\right|+\left\{\int_{0}^{1} M_{q}^{p}\left(\Re^{m} f, r\right)\left(1-r^{2}\right)^{m p} \frac{\varphi^{p}(r)}{1-r} d r\right\}^{\frac{1}{p}} .
$$

Proof. First we consider $m=1$. Then (7) becomes

$$
\|f\|_{p, q, \varphi} \simeq|f(0)|+\left\{\int_{0}^{1} M_{q}^{p}(\Re f, r)\left(1-r^{2}\right)^{p} \frac{\varphi^{p}(r)}{1-r} d r\right\}^{\frac{1}{p}} .
$$

To prove this we notice that, for $f \in H(B)$,

$$
|f(0)| \leq C\|f\|_{p, q, \varphi} \text {. }
$$

Combine [4, p. 80] and 9, Proposition 1.4.7] to have

$$
\left(1-r^{2}\right) M_{q}(\Re f, r) \leq C M_{q}\left(f, \frac{1+r}{2}\right) .
$$

This and the fact that

$$
\varphi(r) \simeq \varphi\left(\frac{1+r}{2}\right)
$$

imply

$$
\left\{\int_{0}^{1} M_{q}^{p}(\Re f, r)(1-r)^{p} \frac{\varphi^{p}(r)}{1-r} d r\right\}^{\frac{1}{p}} \leq C\|f\|_{p, q, \varphi}
$$


Then, by (9) and (11),

$$
\|f\|_{p, q, \varphi} \geq C\left\{|f(0)|+\left\{\int_{0}^{1} M_{p}^{q}(\Re f, r)\left(1-r^{2}\right)^{q} \frac{\varphi^{q}(r)}{1-r} d r\right\}^{\frac{1}{q}}\right\} .
$$

This gives one direction of (8). Notice that

$$
\|f\|_{p, q, \varphi} \leq C\left[\|f(0)\|_{p, q, \varphi}+\|f-f(0)\|_{p, q, \varphi}\right] .
$$

Hence, to prove the other direction of (8) we need only prove

$$
\|f\|_{p, q, \varphi} \leq C\left\{\int_{0}^{1} M_{q}^{p}(\Re f, r)\left(1-r^{2}\right)^{p} \frac{\varphi^{p}(r)}{1-r} d r\right\}^{\frac{1}{p}}
$$

provided $f(0)=0$. For $0<p<\infty$ and $0<q \leq \infty$, (12) can be proved as the estimate (11) in [10, with the only attention that Lemma 1 and Theorem 3 in [10] should be replaced by Lemma 2 in [11] and our Lemma 1 above, respectively. We will omit the details here. Now we deal with the case $p=\infty, 0<q \leq \infty$. If $p=\infty$ and $1 \leq q \leq \infty$, by Lemma 1 and (3) we have

$$
\begin{aligned}
& \sup _{0 \leq r<1} \varphi(r) M_{q}(f, r) \\
\leq & C \sup _{\frac{1}{2} \leq r<1} \varphi(r) M_{q}(f, r) \\
\leq & C \sup _{0 \leq r<1} r \varphi(r) M_{q}(f, r) \\
\leq & C \sup _{0 \leq r<1} \varphi(r) \int_{0}^{r} M_{q}(\Re f, t) d t \\
\leq & C \sup _{0 \leq r<1}(1-r)^{a} \int_{0}^{r} \frac{\varphi(t)}{(1-t)^{a}} M_{q}(\Re f, t) d t \\
\leq & C\left[\sup _{0 \leq r<1}(1-r)^{a} \int_{0}^{r} \frac{d t}{(1-t)^{a+1}}\right] \sup _{0 \leq t<1} \varphi(t)(1-t) M_{q}(\Re f, t) \\
\leq & C \sup _{0 \leq t<1} \varphi(t)(1-t) M_{q}(\Re f, t) .
\end{aligned}
$$

If $p=\infty$ and $0<q<1$, by Lemma 1 and (3) again,

$$
\begin{aligned}
& \sup _{0 \leq r<1} \varphi(r) M_{q}(f, r) \\
\leq & C \sup _{0 \leq r<1} r \varphi(r) M_{q}(f, r) \\
\leq & C \sup _{0 \leq r<1}(1-r)^{a}\left\{\int_{0}^{r} \frac{\varphi(t)^{q}}{(1-t)^{a q}} M_{q}^{q}(\Re f, t) d t\right\}^{\frac{1}{q}} \\
\leq & C\left[\sup _{0 \leq r<1}(1-r)^{a}\left\{\int_{0}^{r} \frac{d t}{(1-t)^{a q+1}}\right\}^{\frac{1}{q}}\right] \sup _{0 \leq t<1} \varphi(t)(1-t) M_{q}(\Re f, t) \\
\leq & C \sup _{0 \leq t<1} \varphi(t)(1-t) M_{q}(\Re f, t) .
\end{aligned}
$$

These give (12) for $p=\infty$ and end the proof of (8).

For general $m$ we see that $(1-r)^{m} \varphi(r)$ is still normal. Then (7) comes from (8) by induction. The proof of the theorem is completed. 


\section{The PRoOF OF THE MAIN RESUlT}

Lemma 2. Given $0<p, q \leq \infty$, take $\beta>b$ and

$$
f_{\zeta}(z)=\frac{\left(1-|\zeta|^{2}\right)^{\beta}}{\varphi(|\zeta|)(1-\langle z, \zeta\rangle)^{\frac{n}{q}+\beta}}, \quad \zeta \in B
$$

Then $\left\|f_{\zeta}\right\|_{p, q, \varphi} \leq C$. Here, the constant $C$ is independent of $\zeta$.

Proof. By [9] we have

$$
M_{q}\left(f_{\zeta}, r\right) \leq C \frac{\left(1-|\zeta|^{2}\right)^{\beta}}{\varphi(|\zeta|)(1-r|\zeta|)^{\beta}} .
$$

Then for $0<p<\infty$,

$$
\begin{aligned}
\left\|f_{\zeta}\right\|_{p, q, \varphi}^{p} & \leq C \int_{0}^{1} \frac{\left(1-|\zeta|^{2}\right)^{p \beta}}{\varphi^{p}(|\zeta|)(1-r|\zeta|)^{p \beta}} \frac{\varphi^{p}(r)}{1-r} d r \\
& =C\left\{\int_{0}^{|\zeta|}+\int_{|\zeta|}^{1}\right\} \frac{\left(1-|\zeta|^{2}\right)^{p \beta}}{\varphi^{p}(|\zeta|)(1-r|\zeta|)^{p \beta}} \frac{\varphi^{p}(r)}{1-r} d r=I_{1}+I_{2} .
\end{aligned}
$$

For $I_{1}$, by (3) and [12] we have

$$
\begin{aligned}
I_{1} & \leq C \frac{(1-|\zeta|)^{p \beta}}{\varphi^{p}(|\zeta|)} \frac{\varphi^{p}(|\zeta|)}{(1-|\zeta|)^{p b}} \int_{0}^{|\zeta|} \frac{(1-r)^{p b-1}}{(1-r|\zeta|)^{p \beta}} d r \\
& \leq C \frac{(1-|\zeta|)^{p \beta}}{(1-|\zeta|)^{p b}} \int_{0}^{1} \frac{(1-r)^{p b-1}}{(1-r|\zeta|)^{p \beta}} d r \\
& \leq C .
\end{aligned}
$$

Similarly,

$$
I_{2} \leq \frac{(1-|\zeta|)^{p \beta}}{(1-|\zeta|)^{p a}} \int_{0}^{1} \frac{(1-r)^{p a-1}}{(1-r|\zeta|)^{p \beta}} d r \leq C
$$

For $p=\infty$,

$$
\left\|f_{\zeta}\right\|_{p, q, \varphi} \leq \sup _{0 \leq r \leq|\zeta|} \varphi(r) M_{q}(f, r)+\sup _{|\zeta| \leq r<1} \varphi(r) M_{q}(f, r)=J_{1}+J_{2} .
$$

For $J_{1}$,

$$
J_{1} \leq C \frac{\left(1-|\zeta|^{2}\right)^{\beta}}{\left(1-|\zeta|^{2}\right)^{b}} \sup _{0 \leq r \leq|\zeta|} \frac{(1-r)^{b}}{(1-r|\zeta|)^{\beta}} \leq C .
$$

Also, $J_{2} \leq C$. Therefore $\left\|f_{\zeta}\right\|_{p, q, \varphi} \leq C$. The lemma is proved.

Proof of Theorem 1. (I). Suppose $g \in H(B)$, and $T_{g}$ is bounded on $H_{p, q}(\varphi)$. First, we claim that, for $f, g \in H(B)$,

$$
\Re\left(T_{g} f\right)(z)=(f \Re g)(z) .
$$


In fact, we may suppose the holomorphic function $f \Re g$ has the expansion $(f \Re g)(z)$ $=\sum_{|\alpha| \geq 1} a_{\alpha} z^{\alpha}$. Then

$$
\begin{aligned}
\Re\left(T_{g} f\right)(z) & =\Re \int_{0}^{1} \sum_{|\alpha| \geq 1} a_{\alpha}(t z)^{\alpha} \frac{d t}{t} \\
& =\Re\left[\sum_{|\alpha| \geq 1} \frac{a_{\alpha} z^{\alpha}}{|\alpha|}\right]=\sum_{|\alpha| \geq 1} a_{\alpha} z^{\alpha} .
\end{aligned}
$$

Notice also that $\left(T_{g} f_{\zeta}\right)(0)=0$. Then for any $\zeta \in B$, by Theorem 1 and Lemma 2,

$$
\begin{aligned}
\left\|T_{g}\right\| & \geq C\left\|T_{g} f_{\zeta}\right\|_{p, q, \varphi} \\
& \geq C\left\{\int_{0}^{1} M_{q}^{p}\left(\Re\left(T_{g} f_{\zeta}\right), r\right)\left(1-r^{2}\right)^{p} \frac{\varphi^{p}(r)}{1-r} d r\right\}^{\frac{1}{p}} \\
& \geq C\left\{\int_{\frac{1+|\zeta|}{2}}^{\frac{3+|\zeta|}{4}} M_{q}^{p}\left(\Re\left(T_{g} f_{\zeta}\right), r\right)\left(1-r^{2}\right)^{p} \frac{\varphi^{p}(r)}{1-r} d r\right\}^{\frac{1}{p}} \\
& \geq C M_{q}\left(\Re\left(T_{g} f_{\zeta}\right), \frac{1+|\zeta|}{2}\right)\left(1-|\zeta|^{2}\right) \varphi(|\zeta|) \\
& \geq C\left|\Re\left(T_{g} f_{\zeta}\right)(\zeta)\right|\left(1-|\zeta|^{2}\right)^{\frac{n}{q}}\left(1-|\zeta|^{2}\right) \varphi(|\zeta|) \\
& =C|\Re g(\zeta)|\left(1-|\zeta|^{2}\right)\left[\left|f_{\zeta}(\zeta)\right|\left(1-|\zeta|^{2}\right)^{\frac{n}{q}} \varphi(|\zeta|)\right] .
\end{aligned}
$$

Hence $g \in \mathcal{B}$ and

$$
\|g\|_{\mathcal{B}} \leq C\left\|T_{g}\right\| .
$$

Conversely, suppose $g \in \mathcal{B}$. Then by Theorem 2 and (14) we get, for any $f \in H(B)$, (17)

$$
\begin{aligned}
\left\|T_{g} f\right\|_{p, q, \varphi} & \leq C\left\{\int_{0}^{1} M_{q}^{p}\left(\Re\left(T_{g} f\right), r\right)\left(1-r^{2}\right)^{p} \frac{\varphi^{p}(r)}{1-r} d r\right\}^{\frac{1}{p}} \\
& =C\left\{\int_{0}^{1} M_{q}^{p}(f, r) \sup \left\{|\Re g(z)|^{p}:|z|=r\right\}\left(1-r^{2}\right)^{p} \frac{\varphi^{p}(r)}{1-r} d r\right\}^{\frac{1}{p}} \\
& \leq C\|g\|_{\mathcal{B}\|f\|_{p, q, \varphi} .}
\end{aligned}
$$

This gives

$$
\|g\|_{\mathcal{B}} \geq C\left\|T_{g}\right\|
$$

(16) and (18) end the proof of (I).

(II). Suppose $T_{g}$ is compact. Since $f_{\zeta}$ weakly convergence to zero in $H_{p, q}(\varphi)$, by the compactness we have as (15)

$$
|\Re g(\zeta)|\left(1-|\zeta|^{2}\right) \leq C\left\|T_{g} f_{\zeta}\right\|_{p, q, \varphi} \rightarrow 0
$$

as $|\zeta| \rightarrow 1$. That is, $g \in \mathcal{B}_{0}$.

Now we let $g \in \mathcal{B}_{0}$. Given any sequence $\left\{f_{m}\right\}$ in $H_{p, q}(\varphi)$ satisfying

$$
\left\|f_{m}\right\|_{p, q, \varphi} \leq 1, \quad f_{m}(z) \rightarrow 0
$$

uniformly on compact subsets of $B$, we are going to prove

$$
\lim _{m \rightarrow \infty}\left\|T\left(f_{m}\right)\right\|_{p, q, \varphi}=0
$$


For any $\varepsilon>0$ we can choose $\eta \in(0,1)$ such that

$$
|\Re g(\zeta)|\left(1-|\zeta|^{2}\right)<\varepsilon \quad \text { if } \eta \leq|z|<1 .
$$

By (17) and (19) we obtain

$$
\begin{aligned}
& \left\|T_{g} f_{m}\right\|_{p, q, \varphi} \leq C\left\{\int_{0}^{1} M_{q}^{p}(f, r) \sup \left\{|\Re g(z)|^{p}:|z|=r\right\}\left(1-r^{2}\right)^{p} \frac{\varphi^{p}(r)}{1-r} d r\right\}^{\frac{1}{p}} \\
& \leq C\left\{\left\{\int_{0}^{\eta} M_{q}^{p}(f, r) \sup \left\{|\Re g(z)|^{p}:|z|=r\right\}\left(1-r^{2}\right)^{p} \frac{\varphi^{p}(r)}{1-r} d r\right\}^{\frac{1}{p}}+\right. \\
& \left.+\left\{\int_{\eta}^{1} M_{q}^{p}(f, r) \sup \left\{|\Re g(z)|^{p}:|z|=r\right\}\left(1-r^{2}\right)^{p} \frac{\varphi^{p}(r)}{1-r} d r\right\}^{\frac{1}{p}}\right\} \\
& \leq C\left\{\|g\|_{\mathcal{B} \sup }\left\{\left|f_{m}(z)\right|:|z| \leq \eta\right\}\right. \\
& \left.+\left\|f_{m}\right\|_{p, q, \varphi} \sup \left\{|\Re g(z)|\left(1-|z|^{2}\right): \eta \leq|z|<1\right\}\right\} \\
& \leq C\left\{\|g\|_{\mathcal{B}} \sup \left\{\left|f_{m}(z)\right|:|z| \leq \eta\right\}+\varepsilon\right\} .
\end{aligned}
$$

Here, the constant $C$ is independent of $f_{m}$ and $\varepsilon$. Therefore, (20) follows. The proof is completed.

\section{ACKNOWLEDGMENT}

This work was done while the author visited the Department of Mathematics at University of North Carolina at Chapel Hill. The author would like to express his thanks to Professor Cima for sharing many ideas. Thanks are also due to the Department of Mathematics at the University of North Carolina at Chapel Hill for their hospitality and to the referee for valuable suggestions.

\section{REFERENCES}

[1] A. Aleman and A. G. Siskakis, An integral operator on $H^{p}$, Complex Variables, 28(1995), 149-158. MR 2000d: 47050

[2] A. Aleman and A. G. Siskakis, Integration operators on Bergman spaces, Indiana University Math. J. 46(1997), 337-356. MR 99b:47039

[3] S. Bochner, Classes of holomorphic functions of several variables in circular domains, Proc. Nat. Acad. Sci. U.S.A. 46(1960), 721-723. MR 22:11144

[4] P. Duren, Theory of $H^{p}$ Spaces, Acad. Press, New York, 1970. MR 42:3552

[5] T. M. Flett, The dual of an inequality of Hardy and Littlewood and some related inequalities, J. Math. Anal. Appl. 38(1972), 746-765. MR 46:3799

[6] G. H. Hardy, Notes on some points in the integral calculus LXVI, Messenger of Math. 58(1929), 50-52.

[7] J. Miao, The Cesàro operator is bounded on $H^{p}$ for $0<p<1$, Proc. Amer. Math. Soc. 116(1992), 1077-1079. MR 93b:47064

[8] Ch. Pommerenke, Schlichte funktionen und analytische funktionen von beschrankter mittler oszilation, Comment. Math. Helv. 52(1977), 122-129. MR 56:12268

[9] W. Rudin, Function Theory in the Unit Ball of $C^{n}$, Springer-Verlag, New York, 1980. MR 82i:32002

[10] J. H. Shi, On the rate of growth of the mean $M_{p}$ of holomorphic and pluriharmonic functions on bounded symmetric domains of $C^{n}$, J. Math. Anal. Appl. 126(1987), 161-175. MR 89d:32011

[11] J. H. Shi and G. P. Ren, Boundedness of the Cesàro operator on mixed norm spaces, Proc. Amer. Math. Soc. 126(1998), 3553-3560. MR 99b:47047

[12] A. L. Shields and D. L. Williams, Bounded projections, duality and multipliers in spaces of analytic functions, Trans. Amer. Math. Soc., 162(1971), 287-302. MR 44:790 
[13] A. G. Siskakis, Composition semigroups and the Cesàro operator on $H^{p}$. J. London Math. Soc. (2) 36 (1987), no. 1, 153-164. MR 89a:47048

[14] R. M. Timoney, Bloch functions in several complex variables. II, J. Reine Angew. Math. 319(1980), 1-22. MR 83b:32005

[15] Z. Xiao, Bergman type spaces and Cesàro operator, Acta Math. Sinica, New Series, 14(1998), 647-654. MR 2001c:46049

Department of Mathematics, Huzhou Teachers College, Huzhou, Zhejiang, 313000 , People's Republic of China - and - Department of Mathematics, University of North Carolina at Chapel Hill, Chapel Hill, North Carolina 27599

E-mail address: huzj@mail.huptt.zj.cn

E-mail address: huz@email.unc.edu 